on the rights of people with mental illness, as well as on fairness, equality and justice. The experience of caring for a family member with mental ill health may bring rewards, but it is also known across countries and cultures to increase the suffering of family members and to heighten their risk of a number of physical, psychological, social and financial problems. These results have led to a call for action at national and international levels to reduce the adverse effects on the families as well as those with lived experience of illness (Gonzalez et al, 2011; World Health Organization, 2013).

Research and future work need to focus on supporting the resilience of patients and families, and on examining their strengths and the factors that protect them. The long-term consequences of caregiving and the support, information and training essential to patients and families at various stages of an illness need to be understood in depth and appropriate empowerment and communication with services formalised. There is, equally, a strong need for mental health professionals to make a thorough assessment of patient and family members' strengths as well as their limitations. Mental health professionals must develop a reliable understanding of the needs of patients for respect, dignity and support during treatment and recovery. They need an equally good understanding of the resources that families have and do not have to support and live successfully with those who are mentally ill.

The continuing support of mental health professionals and policy makers and their positive attitudes towards patient and family participation in turn can give critical support to innovative programmes for patient, family and carer empowerment. Including patients and families as active members of healthcare teams and using their experiences by involving them as trainers and researchers can improve services as well as add support and ease their burdens (Simpson et al, 2002).

\section{References}

Basic Needs (2013) Organizations of Mental Health Service Users and Carers: A Mapping in Sri Lanka Conducted by Basic Needs 2012-13. Basic Needs.

Campbell, J. (2009) Methods. In Handbook of Service User Involvement in Mental Health Research (eds J. Wallcraft, B. Schrank \& M. Amering), pp. 113-138. Wiley-Blackwell.

Collins, P. Y., Patel, V., Joestl, S. S., et al (2011) Grand challenges in global mental health. Nature, 475 (7354), 27-30.

Crawford, M. J., Rutter, D., Manley, C., et al (2002) Systematic review of involving patients in the planning and development of health care. BMJ, 325, 1263-1265.

Dhanda, A. \& Narayan, T. (2007) Mental health and human rights. Lancet, 370, 1198-1199.

Gonzalez, E. W., Polansky, M., Lippa, C. F., et al (2011) Family caregivers at risk: who are they? Issues in Mental Health Nursing $32,528-536$.

Katantoka, S. (2007) Users' networks for Africans with mental disorders. Lancet, 370, 919-920.

Royal Australian and New Zealand College of Psychiatrists (2014) Consumer, Family/Whānau and Carer Engagement, Position Statement 62. Available at https://www.ranzcp.org/Files/Resources/ College_Statements/Position_Statements/PS-62-PPC-Consumerand-Carer-Engagement.aspx (accessed January 2016).

Royal College of Psychiatrists (2009) Mental Health and Social Inclusion, Position Statement PS01/2009. RCPsych.

Simpson, E. L., House, A. O. \& Barkham, M. (2002) A Guide to Involving Users, Ex-users and Carers in Mental Health Service Planning, Delivery or Research: A Health Technology Approach. Academic Unit of Psychiatry and Behavioural Sciences, University of Leeds.

Sunkel, S. (2011) Empowerment and partnership in mental health Lancet, 379, 201-202

Wallcraft, J., Amering, M., Freidin, J., et al (2011) Partnerships for better mental health worldwide: WPA recommendations on best practices in working with service users and family carers. World Psychiatry, 10, 229-236.

World Health Organization (2001) Mental Health: New Understanding, New Hope. World Health Report. WHO

World Health Organization (2013) Mental Health Action Plan 2013-2020. WHO.

\section{THEMATIC} PAPER

\title{
Service users and carers in low- and middle-income countries
}

\author{
Nasser Loza ${ }^{1}$ and Randa Effat ${ }^{2}$
}

${ }^{1}$ The Behman Hospital, Cairo, Egypt, email nloza@behman.com ${ }^{2}$ The Behman Hospital, Cairo, Egypt, email rkeffat@aucegypt.
Taking the Middle East as an example, we explore ways to empower service users and carers. Resources are required for the introduction of community mental health systems and the development of tailor-made psychoeducation, both of which need to be culturally and historically sensitive. A further aim should be the sustained and constructive engagement of families in caring for relatives who have a mental illness, in the community.

\section{Background}

Traditional societies around the world have looked after people who are mentally ill in their communities and families. Some religious cultures believe in the spiritual abilities of individuals with mental illness. It was therefore usual to show kindness and generosity to community members with mental symptoms.

The first psychiatric hospitals in the world were established in the 13th century in the Middle East. Departments of mental health were present in 
general hospitals known as bimaristan in Baghdad, Cairo and Damascus. Qalawoon Hospital in Cairo was built in the 14th century (Jagailloux, 1990).

In the 19th century the emergence of the asylum model of care in low- and middle-income countries (LMICs) encouraged families to abandon their historical responsibility of caring for people who are mentally ill. The practice of separating people with a mental illness from their carers in asylums then spread with Western influence at the end of the 19th century through cultural and political colonisation. Since then, the role of the family and the community in the care of those who are mentally ill has diminished. From our perspective today, it may appear paradoxical that the building of asylums was perceived as one of the benefits of colonisation because it was seen to protect the insane from harm by the public (Mayers, 1982); this suggests that such family care as there was, was not always satisfactory.

In Egypt and other countries in the Middle East, in addition to the stigma associated with mental illness, the practical burden placed on families has contributed to families and communities disowning those who have a mental disorder (Sewilam et al, 2015).

\section{Families and the care of people with mental illness in the Arab world}

The Western model in the middle of the 20th century proposed caring for people who are mentally ill as a joint responsibility of psychologists, physicians, occupational therapists and social workers. However, in the Arab world state-sponsored social welfare has never assumed the importance and diversity of social services in high-income countries. The social distance between members of Arab functionally extended families remains much smaller than that between individuals within typical Western nuclear families (El-Islam, 1989). Moreover, the structural extended family, where members of several generations share the same household, has largely been replaced by a functionally extended family, where sharing and caring continue through frequent and sometimes intense communication between nuclear families within the same clan.

In the Arab world, collaboration between families and professional mental health workers, though diminished, has not broken down completely. Separation of patients with long-standing mental illness from their critical and/or emotionally overinvolved relations, as recommended in the West (Leff \& Vaughn, 1981), has not been practised. El-Islam (2005) affirmed that Arab patients with various types of mental disorder have generally sought and received warmth and positive comments (positive expressed emotions) from relatives. He proposed joint sessions that include patients and family members in psychoeducation, and encouraged family involvement in continued aftercare, while maintaining confidentiality.

According to El-Islam (2005), in Arab cultures the relationship between patient, doctor and family is triangular. Relatives communicate with the doctors before and after seeing the patient, to inform or be informed about the patient's illness. The Arab family is responsible for the care of all its members - both the healthy and the ill. Thus the family is involved in choosing the method of treatment for a relative who is mentally ill. In the Arabian Gulf, first-degree relatives have to approve compulsory hospitalisation of their relatives upon the advice of a psychiatrist, in the absence of mental health legislation. El-Islam (2005) argues that extended families act as 'psychosocial agents', and that this offers a better model than the role typically undertaken by the nuclear family in the rehabilitation of individuals with serious mental disorder.

\section{Service user involvement}

Deinstitutionalisation has been a feature of service provision in many countries over the past 50 years. One aspect of this process is that after patients have been stabilised and discharged following a short hospital stay, families are expected to care for them. It is therefore crucial that the best ways of involving the families and service users themselves in treatment are understood. Kohn (1996) explored the impact on patients of residing in family settings versus in institutions, in light of the carers' understanding of burden and social support. With scarce resources and a limited number of mental health professionals, there remains a need for both psychoeducation to combat stigma associated with mental disorder, and sustained collaboration between families and professionals in the management of serious mental disorder in the Arab world, as elsewhere.

Tambuyzer \& Van Audenhove (2013) suggest that service users, service providers and family carers require further transformation of services. They support the involvement of service users and family carers in taking management decisions, planning and evaluation of care, in cooperation with health providers. Rowe (2012) suggested devising an agreement between mental health institutions and carers, in order to define the role and relationship of each in the care of service users. Involving the family in the treatment plan for their relatives ensures carers' support in aftercare. This team approach has the potential to enhance the quality of mental healthcare services.

\section{The effect of societal change}

Wider changes in the social structures of LMICs have been seen in recent decades. Life expectancy has increased significantly, due to better nutrition and better healthcare. The prevalence of age-related disorders has also increased, particularly the dementias. This has added a burden on services and carers that neither was prepared for. Collaboration between service providers and family carers is developing at a slow pace; as a result, in many countries it has become customary to leave family members with long-standing mental disorders in long-stay institutions. The 
prospect of taking a dementing parent to a home for the elderly still, though, often instils guilt, whereas the medical model provides a culturally acceptable alternative. In many LMICs, the care for individuals with mental disorder is shifting from community-based to institution-based systems.

\section{Studies of carers}

Askey et al (2009) studied the needs of carers for people with psychosis as these disorders present a different set of emotional and physical stressors for carers. The authors differentiated the burden placed upon carers into objective and subjective factors. Objective stress depends on the service user's behaviour. The carer can be affected in several ways, including health or financial consequences, or limitations imposed on daily activities. Subjective burden can be explained by psychological responses, such as 'anxiety, feelings of loss and embarrassment'.

Latoo et al (2010) compared the stress levels of carers for people with schizophrenia, dementia and mood disorders. Carers for people with schizophrenia experienced 'guilt, loss, helplessness, fear, vulnerability, cumulative feelings of defeat, anxiety, resentment and anger'. Caring for those suffering from dementia is challenging; feelings of 'anger, grief, loneliness and resentment' manifest. Mood disorders impose great distress on the carer and the family. Latoo et al (2010) also described how burden can affect family dynamics. They detailed the impact on the carer, whether caring for service users with schizophrenia, dementia or a mood disorder.

To be able to assist carers in supporting their relatives who are mentally ill it is important to identify their needs. Askey et al (2009) recommended that professionals look out not only for the needs of the service users but for those of the carer as well - by addressing carers' need for knowledge about the illness their relatives have, as well as the needs for emotional and financial support. Stanbridge et al (2013) pointed out the importance of recognising the efforts of families and carers in caring for the elderly. McAndrew \& Wilkinson (2008) acknowledged the difficulty of caring for service users with unpredictable odd behaviours.

Carers often develop physical and mental problems. Latoo et al (2010) suggested some coping techniques for carers to lessen the psychological stress; they describe cognitive-behavioural family interventions, educational and emotional support, and spiritual help.

\section{Conclusion}

Looking after a close family member with a mental illness can be overwhelming and emotionally draining. There is a global call for carers to be involved in caring for their relatives. The process of accepting this model of collaboration has been slow to develop in LMICs. Often carers are perceived 'as part of the problem' (Rowe, 2012, p. 22). This is partly due to the lack of information regarding the aetiology of mental illness. The stigma that is often attached to illnesses such as schizophrenia, dementia and other psychological disorders hampers the carer's role. This stigma has contributed to carers' sense of isolation from professionals in the field of mental health and from the community in general. The involvement of service users and their families is increasingly considered a valuable approach that is beneficial in enhancing the quality of mental healthcare services.

\section{References}

Askey, R., Gamble, G., Gray, R., et al (2009) What do carers of people with psychosis need from mental health services? Exploring the views of carers, service users and professionals. Journal of Family Therapy, 31(3), 310-331.

El-Islam, M. F. (1989) Collaboration with families for the rehabilitation of schizophrenic patients and the concept of expressed emotions. Acta Psychiatrica Scandinavica, 79, 303-309.

El-Islam, M. F. (2005) Some cultural aspects of the Arab patientdoctor relationship. International Psychiatry, 7, 18-20.

Jagailloux, S. (1990) La médicalisation de l'Egypte au xix siècle (1798-1918). Revue française d'histoire d'outre-mer année, 77(286), 123-124

Kohn, L. P. (1996) Familial caretaking in rural families: characteristics in caring for the seriously mentally ill, pp. 3-12. Dissertation presented to the Graduate Faculty of the University of Virginia.

Latoo, J., Wadoo, O. \& Shah, A. J. (2010) Psychological distress in carers of people with mental disorders. British Journal of Medical Practitioners, 3(3), a327.

Leff, J. P. \& Vaughn, C. F. (1981) The role of maintenance therapy and relatives' expressed emotion in the relapse of schizophrenia: a two-year follow-up. British Journal of Psychiatry, 139, 102-104.

Mayers, M. (1982) A century of psychiatry: the Egyptian mental hospital. PhD dissertation, Princeton University.

McAndrew, S. \& Wilkinson, C. (2008) 'I'm not an outsider, I'm his mother!' A phenomenological enquiry into carer experiences of exultation from acute psychiatric settings. International Journal of Mental Health Nursing, 17, 392-401.

Rowe, J. (2012) A covenant between mental health services and family carers. Mental Health Practice, 2, 22-23.

Sewilam, A. M., Watson, A. M., Kassem, A. M., et al (2015) Suggested avenues to reduce the stigma of mental illness in the Middle East. International Journal of Social Psychiatry, 61(2), 111-120.

Stanbridge, R. I., Burbach, F. R., Rapsey, E. H., et al (2013) Improving partnerships with families and carers in in-patient mental health services for older people: a staff training programme and family liaison service. Journal of Family Therapy, 35(2), 176-197.

Tambuyzer, E. \& Van Audenhove, C. (2013) Service user and family carer involvement in mental health care: divergent views. Community Mental Health Journal, 49(6), 675-685. 